\title{
Estudio teórico de las propiedades estructurales y electrónicas en sistemas $\mathrm{BaTiO}_{3}$ y $\mathrm{BaZrO}_{3}$
}

\section{Theoretical study of structural and electronic properties in $\mathrm{BaTiO}_{3}$ and $\mathrm{BaZrO}_{3} \mathrm{Systems}$}

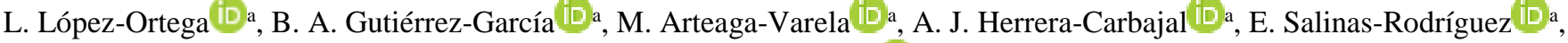 \\ V. Rodríguez-Lugo iD a* \\ a Área Académica de Ciencias de la Tierra y Materiales, Instituto de Ciencias Básicas e Ingeniería, Universidad Autónoma del Estado de Hidalgo, Carretera Pachuca-
} Tulancingo Km. $4.5,42184 .^{1}$

\section{Resumen}

Se realizó un estudio teórico sobre dos minerales de perovskita de tipo $\mathrm{ABO}_{3}$, Titanato de Bario $(\mathrm{BaTiO})_{3}$ y $\mathrm{Zirconato}$ de Bario $\left(\mathrm{BaZrO}_{3}\right)$, optimizando los parámetros para un pseudopotencial Perdew-Burke-Erszerhof (PBE) de tipo ultrasuave, mediante el uso del código SIESTA el cual está sustentado en la Teoría del Funcional de la Densidad (DFT). Así mismo se optimizaron las estructuras, alterando el parámetro de red de ambas perovskitas permitiendo determinar la más estable (mínima energía). Para las estructuras de mínima energía del $\mathrm{BaTiO}_{3}$ y $\mathrm{BaZrO}_{3}$ se obtuvieron parámetros de red correspondientes a $4.104 \AA$ y $4.276 \AA$ respectivamente, que difieren en poca cantidad a los reportados experimentalmente cuyos valores son de $4.004 \AA$ y $4.256 \AA$ mostrando que SIESTA permite obtener aproximaciones superiores al 97\%, asimismo la estructura de bandas mostro el comportamiento indirecto en la transición de la banda de valencia a la de conducción para ambos materiales con características de semiconductores al poseer valores de 1.64 y 2.77 eV respectivamente.

\section{Palabras Clave:}

$\mathrm{BaTiO}_{3}, \mathrm{BaZrO}_{3}, \mathrm{DFT}$, Optimización, Pseudopotencial, Perovskita.

\begin{abstract}
A theoretical study was carried out on two $\mathrm{ABO}_{3}$ type perovskite minerals, Barium Titanate $\left(\mathrm{BaTiO}_{3}\right)$ and $\mathrm{Barium} \mathrm{Zirconate}(\mathrm{BaZrO})$. The parameters were optimized for a Perdew-Burke-Erszerhof (PBE) pseudopotential of the ultrasoft type, by using the SIESTA code, which is supported by the Density Functional Theory (DFT). The structures were then optimized, altering the lattice parameter of both perovskites allowing the most stable (minimum energy) to be determined. For the minimum energy structures of $\mathrm{BaTiO}_{3}$ and $\mathrm{BaZrO}_{3}$, lattice parameters corresponding to $4.104 \AA$ and $4.306 \AA$ respectively were obtained, which differ slightly from those reported experimentally, whose values are $4.004 \AA$ and $4.276 \AA$, showing that SIESTA allows obtaining approximations higher than $97 \%$, also the band structure showed the indirect behavior in the transition from the valence band to the conduction band for both materials with semiconductor characteristics, having values of 1.64 and $2.77 \mathrm{eV}$ respectively..
\end{abstract}

Keywords:

$\mathrm{BaTiO}_{3}, \mathrm{BaZrO}_{3}$, DFT, Optimization, Pseudopotential, Perovskite.

\section{Introducción}

Actualmente en nuestra sociedad el uso de materiales avanzados es una de las principales necesidades debido a las constantes demandas tecnológicas e industriales las cuales no pueden sustentar los materiales usados actualmente (Mijangos y Serafín, 2007), para ello la ciencia de los materiales focaliza su esfuerzo en la innovación de estos así mismo permitiendo el desarrollo de nuevas técnicas computacionales basadas en diferentes teorías que permitan tratar con mayor precisión la interacción entre átomos, iones y/o moléculas de algunos sólidos.

Una de esas teorías es la Teoría de Funcionales de la Densidad (DFT por sus siglas en inglés), que es un procedimiento variacional alternativo a la solución de la ecuación de Schrödinger. Es uno de los métodos más utilizados en los cálculos cuánticos de la estructura electrónica de la materia (Klaus, 2006). Esta teoría provee de un método alternativo y riguroso que permite

\footnotetext{
*Autor para la correspondencia: ventura.r165@gmail.com

Correo electrónico: leonardolp60@ gmail.com (Leonardo López-Ortega), bray5032000@gmail.com (Brayann Aldair Gutiérrez-García), miguelnano1@gmail.com (Miguel ArteagaVarela), alejandro_herrera@uaeh.edu.mx (Alejandro de Jesús Herrera-Carbajal), salinasr@uaeh.edu.mx (Eleazar Salinas-Rodríguez), ventura.rl65@gmail.com (Ventura RodríguezLugo) 
calcular el estado fundamental de estructuras complejas como lo son las estructuras de tipo $\mathrm{ABO}_{3}$.

Los sólidos de estructura cristalina tipo $\mathrm{ABO}_{3}$, mejor conocidos como perovskitas presentan propiedades de gran interés científico y tecnológico debido a que sus estructuras electrónicas las dotan de distintos tipos de propiedades, por ello pueden llegar a ser aislantes o conductores eléctricos, además de semiconductores o superconductores. La estructura perovskita es capaz de adaptarse a un enorme número de combinaciones de cationes A y B mediante las distorsiones en la orientación de la red de octaedros y mediante distorsiones dentro de los mismos octaedros (Blanco, Fuertes y Carbonio, 2015). De esta manera las perovskitas adoptan una amplia gama de propiedades multifuncionales captando así la atención científica debido a que se ha demostrado que es un excelente material avanzado.

Por tal motivo, el presente trabajo tiene como propósito el estudio de las estructuras de Titanato de Bario $\left(\mathrm{BaTiO}_{3}\right)$ y Zirconato de Bario $\left(\mathrm{BaZrO}_{3}\right)$, siendo que el $\mathrm{BaTiO}_{3}$ es uno de los materiales electro-cerámicos más clásicos al ser en sí, el primer material ferroeléctrico-cerámico desarrollo, presentando propiedades dieléctricas, ferroeléctricas y fotorretractivas que la posicionan como un material fundamental en la fabricación de condensadores cerámicos multicapa (Caballero, Fernández, Durán y Moure, 1994). El $\mathrm{BaTiO}_{3}$ presenta una estructura cristalina similar al Titanato de Calcio $\left(\mathrm{CaTiO}_{3}\right)$ de tipo $\mathrm{ABO}_{3}$. Esta red cristalina es adoptada por múltiples compuestos de la forma $\mathrm{ABO}_{3}$ que en el caso del $\mathrm{BaTiO}_{3}$, la simetría cúbica es estable por encima de $130^{\circ} \mathrm{C}$ hasta temperaturas muy altas (aproximadamente $1400^{\circ} \mathrm{C}$ ) en las que aparece la fase hexagonal. Por debajo de $130^{\circ} \mathrm{C}$ y hasta $0^{\circ} \mathrm{C}$ tienen simetría tetragonal, entre $0^{\circ} \mathrm{C} \mathrm{y}-88^{\circ} \mathrm{C}$ pasa a ser ortorrómbica y a temperaturas inferiores romboédrica (Bunget y Popescu, 1984).

En el caso de la perovskita $\mathrm{BaZrO}_{3}$, es un mineral conocido por su alta estabilidad química y excelente conductividad de protones cuando es dopado, normalmente con Itrio, lo que hace que el $\mathrm{BaZrO}_{3}$ tras más de 30 años de estudio, sea considerado como uno de los materiales más prometedores para aplicaciones en electrolizadores, celdas de combustible o celdas de conversión de metano. También posee un amplio rango de temperaturas de fusión alrededor de $2600^{\circ} \mathrm{C}$, los cual hace que exhiba una alta resistencia a los límites de grano que influye directamente en su rendimiento eléctrico, generando así bajos coeficientes de expansión térmica y baja conductividad térmica. (Bausá, 2020)

Sin embargo, los estudios de las propiedades estructurales y electrónicas sobre estos dos perovskitas se centran en investigaciones experimentales, y pocos son los proyectos teóricos basados en la simulación molecular, de esta manera la importancia de los estudios teóricos de los materiales que permiten explicar el comportamiento de los mismos a diferentes estímulos. Los anterior contribuye al acceso al modelado de estructuras nuevas que son complejas para su desarrollo en forma experimental, lo que favorece la obtención de datos previos para realizar una optimización de los materiales empleados en la creación de estructuras complejas, por otra parte, ayuda a complementar los estudios realizados de forma experimental, a través de la comparación de los datos obtenidos mediante ambas rutas.

Por consiguiente, se realizó un estudio teórico mediante simulación molecular a primeros principios, con el fin de establecer la estructura más estable (de mínima energía) para las perovskitas de $\mathrm{BaTiO}_{3}$ y $\mathrm{BaZrO}_{3}$ determinando la energía de Fermi, la estructura de bandas y densidad de estados para conocer el comportamiento electrónico del material en bulto.

\section{Metodología}

En este trabajo se realizaron cálculos de primeros principios de propiedades estructurales y electrónicas específicamente, se calculó la estructura de bandas y densidad de estados electrónica de los sistemas $\mathrm{BaTiO}_{3}$ y $\mathrm{BaZrO}_{3}$, mediante la solución de las ecuaciones mecánico-cuánticas usando la aproximación de la teoría del funcional de la densidad (DFT), dentro del marco de la aproximación de gradiente generalizado (GGA) en la parametrización de Perdew, Burke y Ernzerhof (PBE), a través de la implementación del código SIESTA mediante el uso de orbitales y pseudopotenciales atómicos ultra suaves.

Para comprender los principios sobre los que funciona este estudio teórico primero se necesita entender los fenómenos cuánticos que están presentes en las partículas. El estado físico de mínima energía de una partícula o un sistema de partículas se representa mediante la función de onda de Schrödinger independiente del tiempo (David j. Griffiths, 2004) expresada en la ecuación 1:

$$
i \hbar \frac{\partial}{\partial t} \Psi(\mathrm{r})=\hat{\mathrm{H}} \Psi(\mathrm{r})
$$

Para un sistema multielectrónico está dado por la ecuación 2:

$$
i \hbar \frac{\partial}{\partial t} \Psi\left(r_{1}, r_{2}, \ldots r_{N}\right)=\hat{\mathrm{H}} \Psi\left(r_{1}, r_{2}, \ldots r_{N}\right)
$$

Donde $\hat{\mathrm{H}}$ es el operador Hamiltoniano que representa la contribución de energía cinética y potencial del sistema. Sin embargo, uno de los principales problemas en la teoría cuántica de muchos cuerpos es encontrar una solución exacta a la ecuación de Schrödinger, no obstante, existen muchos métodos que se han desarrollado para resolver este problema a partir de aproximaciones en el hamiltoniano para que adquiera una forma más simple. Entre ellos encontramos el desarrollado por HartreeFock, la aproximación de Born-Oppenheimer y la desarrollada por Hohenberg-Kohn.

La base de la teoría de DFT se centra en la aproximación adiabática y en los postulados de Hohenberg y Kohn desarrollados en 1964 (Hohenberg \& Kohn, 1964) donde mostraron que a partir de la densidad electrónica se puede minimizar el funcional de energía del sistema como se aprecia en la ecuación 3:

$$
E[p]=F[p]+\int p(r) v(r) d r
$$

donde $\int p(r) v(r) d r$ es un potencial externo y $\mathrm{F}[\mathrm{p}]$ viene dado por:

$$
F[n(r)]=T_{s}[n(r)]+E_{e-e}[n(r)]+V_{x c}[n(r)]
$$

También, existe una representación a la aproximación del funcional universal que es la interacción electrón-electrón que se propone como parte principal de la interacción coulómbica dada por la ecuación 5:

$$
J[p]=\frac{1}{2} \iint \frac{p(r) p\left(r^{\prime}\right)}{\left|r-r^{\prime}\right|} d r d r^{\prime}
$$


Y con esto, el funcional universal se escribe como:

$$
F[p]=T[p]+V_{e e}[p]=T_{s}[p]+J[p]+E_{x c}[p]
$$

Entonces los orbitales de Kohn-Sham son aquellos que satisfagan las ecuaciones integro-diferenciales expresados en la ecuación 7 :

$$
\left(-\frac{1}{2} \nabla^{2}+v_{e f f}(r)\right) \emptyset_{i}(r)=\varepsilon_{i} \emptyset_{i}(r)
$$

La densidad de estados (DOS) se define como la distribución cuya integral entre dos energías da el número existente de estados, donde su expresión matemática se representa en la ecuación 8:

$$
d N(E)=D(E) d(E)=N\left(E_{2}\right)-N\left(E_{1}\right)=\int_{E_{1}}^{E_{2}} D(E) d(E)
$$

Dicho lo anterior, podemos agregar que el esquema metodológico central de SIESTA consiste en combinar orbitales pseudoatómicos de soporte finito como conjuntos de bases, pseudopotenciales que conservan normas y una cuadrícula de espacio real para la representación de la densidad de carga y los potenciales y el cálculo de sus elementos de matriz asociados (Villa Ortega, 2007).

Este estudio teórico se divide en 3 partes donde, primeramente, se modelarán las estructuras pertenecientes a los grupos $\mathrm{ABO}_{3}$ en su representación más simple para conocer los parámetros reticulares y llevar a cabo un proceso de relajamiento a fin de encontrar la estructura de mínima energía. Finalmente se obtendrán la densidad de estado de las estructuras centrosimétricas y relajadas de ambas perovskitas para poder determinar los cambios que pudieron llevarse a cabo en los orbitales moleculares de valencia de cada uno de los átomos de la red para determinar qué tipo de propiedades presentará mediante el band gap.

En la primera parte del estudio se ajustaron el tamaño de la función de onda de prueba mediante movimiento rígido de la estructura con el fin de disminuir la variación entre las fuerzas interatómicas del sistema en bulto en 0.1 órdenes de magnitud.

Los puntos de alta simetría en el espacio recíproco fueron ajustados para $\mathrm{BaTiO}_{3}$ y $\mathrm{BaZrO}_{3}$ empleando el método de mallas Monkhorst-Pack (MP), el cual emplea vectores primitivos en el espacio reciproco que están definidos a partir de los vectores primitivos en el espacio real, en la Tabla 1 y 2 correspondientes al ajuste del $\mathrm{BaTiO}_{3}$ y $\mathrm{BaZrO}_{3}$, donde la sección remarcada representa el valor de mínima energía que corresponde a la red Monkhorst-Pack.

Tabla 1: Ajuste de puntos de alta simetría (puntos $K$ ) en la primera zona de Brillouin de la red recíproca en función de la energía total del sistema del $\mathrm{BaTiO}_{3}$

\begin{tabular}{cccc}
\hline $\begin{array}{c}\text { Energía total } \\
(\mathrm{eV})\end{array}$ & $\begin{array}{c}\text { Ecutwfc } \\
(\mathrm{Ry})\end{array}$ & $\begin{array}{c}\text { No. de } \\
\text { puntos } \mathrm{k}\end{array}$ & $\begin{array}{c}\text { Red } \\
\text { MP }\end{array}$ \\
\hline-24.6721 & 300 & 4 & $3 \times 3 \times 3$ \\
-24.6480 & 300 & 10 & $5 \times 5 \times 5$ \\
-24.6445 & 300 & 20 & $7 \times 7 \times 7$ \\
-24.6439 & 300 & 35 & $9 \times 9 \times 9$ \\
\hline
\end{tabular}

De los resultados obtenidos anteriormente gracias al ajuste de los puntos de alta simetría en el espacio recíproco, se tomó como valor óptimo la malla de $7 \times 7 \times 7$ en la cual hay 20 puntos $\mathrm{k}$ ya que la variación de la energía total del sistema para las mallas consecutivas tiene una muy ligera variación.

Tabla 2: Ajuste de puntos de alta simetría (puntos $K$ ) en la primera zona de Brillouin de la red recíproca en función de la energía total del sistema del $\mathrm{BaZrO}_{3}$

\begin{tabular}{cccc}
\hline $\begin{array}{c}\text { Energía total } \\
(\mathrm{eV})\end{array}$ & $\begin{array}{c}\text { Ecutwfc } \\
(\mathrm{Ry})\end{array}$ & $\begin{array}{c}\text { No. de } \\
\text { puntos k }\end{array}$ & $\begin{array}{c}\text { Red } \\
\text { MP }\end{array}$ \\
\hline-23.4527 & 160 & 4 & $3 \times 3 \times 3$ \\
-23.4302 & 160 & 10 & $5 \times 5 \times 5$ \\
-23.4284 & 160 & 20 & $7 \times 7 \times 7$ \\
-23.4282 & 160 & 35 & $9 \times 9 \times 9$ \\
\hline
\end{tabular}

Así mismo, de los resultados obtenidos anteriormente gracias al ajuste de los puntos de alta simetría en el espacio recíproco, también se tomó como valor óptimo la malla de $7 \times 7 \times 7$ en la cual hay 20 puntos k ya que la variación de la energía total del sistema para las mallas consecutivas tiene una muy ligera variación.

En la tercera parte del estudio se calcula la densidad electrónica $[n(r)]$ de las estructuras basales, empleando un algoritmo BFGS (Broyden-Fletcher-Goldfarb-Shanno) se optimizaron las posiciones atómicas y los parámetros de red para encontrar la configuración de mínima energía (mayor estabilidad); aunado a esto se procede a la determinación de la estructura de bandas y densidad de estados electrónicos permitiendo conocer el comportamiento electrónico de los sistemas en bulto.

En particular en este estudio se definió una ruta $\Gamma-\mathrm{X}-\mathrm{M}-\Gamma-\mathrm{R}$ $\mathrm{X}$ en la primera zona de Brillouin en el espacio recíproco (Arteaga Varela, 2019) para calcular la estructura de bandas y densidad de estados, en la Tabla 3 se muestran las coordenadas de los puntos de alta simetría correspondientes a la ruta mencionada con anterioridad.

Tabla 3: Puntos de alta simetría en la red recíproca para $\mathrm{BaTiO}_{3}$ y $\mathrm{BaZrO}_{3}$

\begin{tabular}{c|ccc}
\hline Punto & \multicolumn{3}{c}{$\begin{array}{c}\text { Coordenadas cartesianas en el espacio } \\
\text { recíproco }\end{array}$} \\
\hline$\Gamma$ & 0.000 & 0.000 & 0.000 \\
$\mathrm{X}$ & 1.000 & 0.000 & 0.000 \\
$\mathrm{M}$ & 1.000 & 1.000 & 0.000 \\
$\Gamma$ & 0.000 & 0.000 & 0.000 \\
$\mathrm{R}$ & 1.000 & 1.000 & 1.000 \\
$\mathrm{X}$ & 1.000 & 0.000 & 0.000 \\
\hline
\end{tabular}

\section{Resultados y discusiones}

En este estudio teórico mediante el uso del código SIESTA que se basa en la teoría del funcional de la densidad con base principal de orbitales atómicos y utilizando un pseudopotencial de aproximación de gradiente generalizado se llevó a cabo un procedimiento metodológico a fin de optimizar la geométrica de las estructuras de $\mathrm{BaTiO}_{3}$ y $\mathrm{BaZrO}_{3}$ respectivamente. En la Figura 1 se muestran las estructuras cristalinas de los sistemas mencionados anteriormente. 


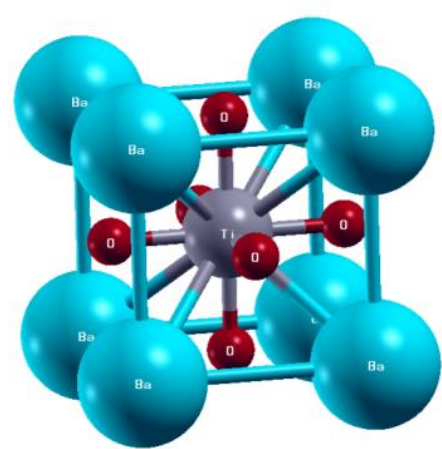

a)

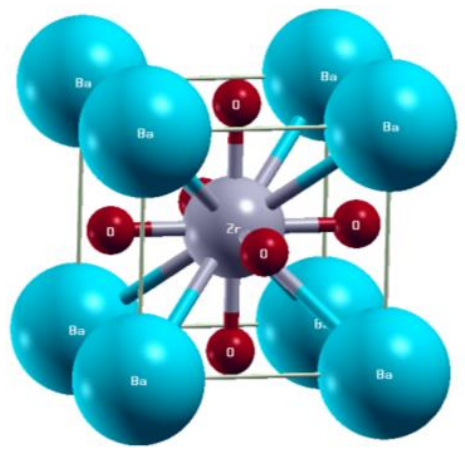

b)

Figura 1: Representación 3D de la estructura cristalina de a) $\mathrm{BaTiO}_{3}$ y b) $\mathrm{BaZrO}_{3}$ generados por Xcrysden.

Se realizó un ajuste en los parámetros de red para la determinación del sistema de equilibrio (mínima energía) para ambas perovskitas, los resultados se presentan en la Tabla 4. Las secciones remarcadas representan el valor del sistema de mínima energía.

Tabla 4: Optimización estructural del $\mathrm{BaTiO}_{3}$ con variación de parámetros de red en (lado izquierdo) 0.1 y (lado derecho) 0.01 respectivamente

\begin{tabular}{cccc}
\hline $\begin{array}{c}\text { Parámetro } \\
\text { de red }\end{array}$ & $\begin{array}{c}\text { Energía total } \\
(\mathrm{eV})\end{array}$ & $\begin{array}{c}\text { Parámetro } \\
\text { de red }\end{array}$ & $\begin{array}{c}\text { Energía total } \\
(\mathrm{eV})\end{array}$ \\
\hline 3.804 & -1617.86600 & 4.094 & -1619.81681 \\
3.904 & -1619.07826 & 4.104 & -1619.81922 \\
4.004 & -1619.66084 & 4.114 & -1619.81860 \\
4.104 & -1619.81922 & 4.124 & -1619.81527 \\
4.204 & -1619.71198 & 4.134 & -1619.80886 \\
& & 4.144 & -1619.80037 \\
& & 4.154 & -1619.79113 \\
& & 4.164 & -1619.78034 \\
& & 4.174 & -1619.76726 \\
& & 4.184 & -1619.75131 \\
& & 4.194 & -1619.73273 \\
& & 4.204 & -1619.71198 \\
\hline
\end{tabular}

Como se muestra en la Tabla 4, se variaron los parámetros de red en 0.1 angstrom (izquierda) y de 0.01 (derecha) para encontrar la estructura más estable, se utilizó como valor de mínima energía el parámetro reticular de $4.104 \AA$ A la cual posee una aproximación del $97.5 \%$ al valor reportado experimentalmente de $4.004 \AA$.
Para el sistema zirconato de bario, los valores del ajuste para la determinación de la estructura en equilibrio se presentan en la Tabla 5.

Tabla 5: Optimización estructural del $\mathrm{BaZrO}_{3}$ con variación de parámetros de red en (lado izquierdo) 0.1 y (lado derecho) 0.01 respectivamente

\begin{tabular}{cccc}
\hline $\begin{array}{c}\text { Parámetro } \\
\text { de red }\end{array}$ & $\begin{array}{c}\text { Energía total } \\
(\mathrm{eV})\end{array}$ & $\begin{array}{c}\text { Parámetro } \\
\text { de red }\end{array}$ & $\begin{array}{c}\text { Energía total } \\
(\mathrm{eV})\end{array}$ \\
\hline 4.156 & -1613.0687 & 4.266 & -1613.2933 \\
4.256 & -1613.2878 & 4.276 & -1613.2944 \\
4.356 & -1613.1637 & 4.286 & -1613.2912 \\
4.456 & -1612.7149 & 4.296 & -1613.2841 \\
& & 4.316 & -1613.2579 \\
& & 4.326 & -1613.2391 \\
& & 4.336 & -1613.2172 \\
& & 4.346 & -1613.1918 \\
& & 4.356 & -1613.1637 \\
& & 4.366 & -1613.1305 \\
\hline
\end{tabular}

Como se observa para este sistema, se ocupó la misma variación del parámetro de red para determinación del estado basal del sistema $\mathrm{BaZrO}_{3}$; se obtuvo como valor de mínima energía el parámetro reticular de $4.276 \AA$, el cual posee una aproximación del $99.5 \%$ al valor reportado experimentalmente de $4.256 \AA$.

En la Figura 2 se observa de forma gráfica los valores presentados en las tablas anteriores, donde se puede apreciar los valores de mínima energía en los sistemas de estudio.

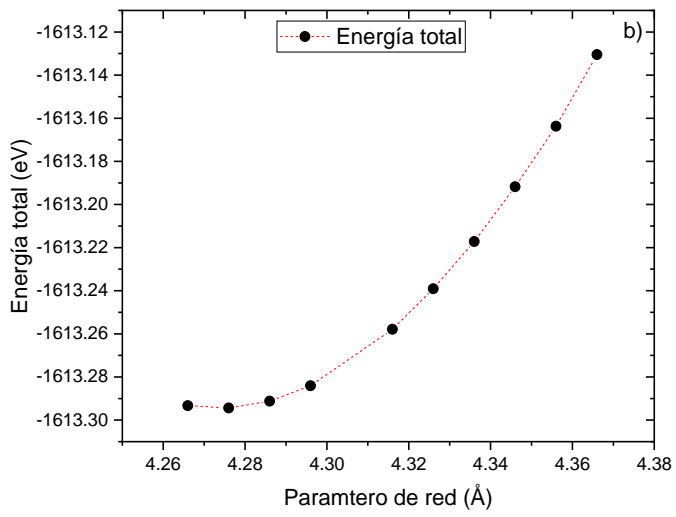

Figura 2: Parámetro de red contra energía total del sistema de a) $\mathrm{BaTiO}_{3}$ y b) $\mathrm{BaZrO}_{3}$ 

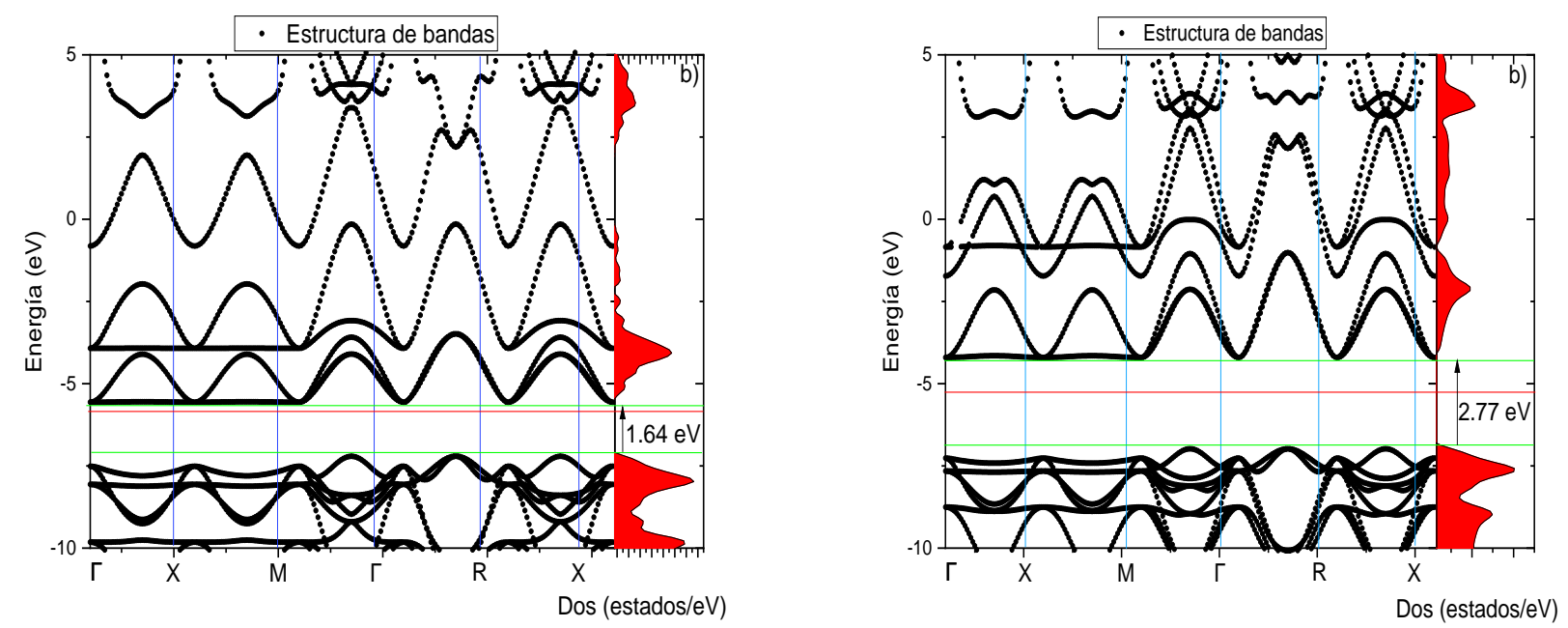

Figura 3: Diagrama de dispersión electrónica de bandas y densidad de estados para a) $\mathrm{BaTiO}_{3}$ y b) $\mathrm{BaZrO}_{3}$

En la Figura 3a se observa que el material es de brecha indirecta dado que el punto de transición en la banda de valencia se encuentra en la posición más cercana a $\mathrm{R}$, y el punto de transición en la banda de conducción se encuentra en $\Gamma$. Asimismo, se obtuvo una magnitud de bandgap de $1.64 \mathrm{eV}$ para el titanato de bario, la cual difiere del valor experimental y corresponde a $2.9 \mathrm{eV}$ (Gomathi \& Nithya, 2017), lo que es correcto al emplear el código SIESTA debido a que subestima los valores obtenidos para las transiciones electrónicas determinadas por la estructura de bandas. La densidad de estados presentada a un costado de la estructura de bandas corrobora la amplitud de la banda; también proporciona información numérica sobre la disponibilidad de los estados en cada nivel de energía. Un valor alto en la densidad de estados representa un número alto de estados disponibles a cierto valor de energía, por esto si no hay estados disponibles para la ocupación como en el caso del bandgap, el valor de los estados será cero.

Para la Figura 3b la transición entre las bandas tiene un comportamiento similar al anterior, sin embargo, el valor de la transición indirecta corresponde a $2.77 \mathrm{eV}$. De igual forma, la densidad de estados corrobora la amplitud de la banda prohibida y el cual difiere del reportado experimentalmente, cuyo valor corresponde a $5 \mathrm{eV}$ (Heifets \& Merinov, 2007).

Para ambos materiales se encuentra un comportamiento semiconductor correspondiente al valor obtenido en su estructura de bandas, sin embargo, al poseer una transición indirecta, el material tiende a oponer mayor resistencia para provocar la transición de los electrones de la banda de valencia a la de conducción. Esto indica que durante el proceso de recombinación de los pares hueco-electrón se necesita otra energía en forma de fonones para que el electrón cambie de momento a donde se encuentra el punto mínimo de la banda de conducción (LUMO), por lo que la energía determinada en las gráficas no corresponde a la energía total para provocar dicho fenómeno de transición electrónica. Por ello, realizar el estudio de la parte imaginaria del tensor dieléctrico permitiría conocer el valor correspondiente al mínimo de la transición directa entre ambas bandas.

$\mathrm{El}$ analizar dos sistemas similares como el $\mathrm{BaTiO}_{3}$ y $\mathrm{BaZrO}_{3}$ con la misma fase cristalina, pero con solo un ion diferente en su estructura permite entablar una comparación del comportamiento electrónico al presentar una mayor cantidad de electrones interactuantes un sistema con relación al otro. En este caso el Zirconato de Bario posee una mayor cantidad de electrones que generan diferente cantidad de contribuciones electrónicas.

Las contribuciones electrónicas de los electrones adicionales que posee el ion $\mathrm{Zr}$ con respecto al ion $\mathrm{Ti}$, permite entender que la interacción de un ion diferente en un sistema, modifica las propiedades que posee dicho material de estudio en un sistema en bulto. Asimismo, en ambos sistemas se exhibe una subestimación de la banda prohibida cercano al 56\% del valor experimental. Códigos como SIESTA subestiman los valores de la bandgap para sistemas en bulto (Soler, 2002).

Los comportamientos resultantes en la optimización geométrica, al igual que los pertenecientes a las propiedades electrónicas, son atribuidos al tipo de pseudopotencial con que se trabajó. Estudios previos realizados empleando código wienk $2 \mathrm{k}$ basado en onda plana aumentada lineal de potencial completo (FPLAPW por sus siglas en inglés) muestran un comportamiento indirecto en ambos materiales, con valores para el $\mathrm{BaTiO}_{3}$ y $\mathrm{BaZrO}_{3}$ de 1.2 y 2.2 eV respectivamente (Khenata, 2005), indicando que en el presente estudio se obtuvieron valores con una mayor aproximación para ambos sistemas cristalinos. De igual forma, para generar una aproximación con mayor grado de precisión para ambas estructuras en bulto, se puede efectuar la fabricación de nuevos pseudopotenciales que el código permita emplear o la implementación de potenciales híbridos que permitan generar un mejor ajuste en los resultados generados por el código.

\section{Conclusiones}

Se observó que la energía total de los sistemas converge en relación al parámetro de red y a las posiciones atómicas, reproduciéndose con un error relativo cercano a $2.5 \%$. Asimismo, la forma de la estructura de bandas y su respectiva densidad de estados electrónica para ambos sistemas es similar y coincide con estudios previos reportados con un pseudopotencial distinto, las cuales muestran un comportamiento semejante. Por lo tanto, se puede inferir que el pseudopotencial que se utilizó en este estudio reproduce bien las propiedades estructurales y algunas propiedades electrónicas para estos materiales. 
En conclusión, el uso de este tipo de técnicas es útil para calcular propiedades de materiales como su estructura cristalina o la estabilidad con respecto a su fase, estructura de bandas (valor del bandgap y tipo de banda), entre otras; información que se puede utilizar como herramienta para el diseño de materiales.

Ambos materiales al tener un carácter indirecto en la transición electrónica, pueden ser empleados para el desarrollo de capacitores para circuitos electrónicos, por lo cual se recomienda el estudio de propiedades ópticas y magnéticas para su posible aplicación en el diseño de estructuras metamateriales.

\section{Referencias}

Bernal Carrillo, Lina. (2016). Influencia de los parámetros de síntesis y deposición en la elaboración y eficiencia de celdas solares de perovskita y su caracterización por métodos electroquímicos y de superficie. 2020, de Univerisdad de los Andes Sitio web: https://repositorio.uniandes.edu.co/bitstream/handle/1992/18974/u728490 .pdf? sequence $=1$ \&isAllowed $=y$

Cortés, M.R.S. Propiedades estructurales y electrónicas del cátodo Lí (MnyFe1-y) P04 mediante primeros principios, Universidad Nacional de Colombia, 2013.

Volume16, Issue12, December 1989, Pages 1399-1402, https://doi.org/10.1029/GL016i012p01399

Volume 462, Issues 1-3, 10 August 2000, Pages 19-35, https://doi.org/10.1016/S0039-6028(00)00603-8

Anderson OL, Liebermann RC (1970) Equations for the elastic constants and their pressure derivatives for three cubic lattices and some geophysical applications. Phys Earth Planet Inter 3:61-85

E.E. Rozo A. a, F. Fajardo b , D.A. Landinez Tellez a , J. Arbey Rodríguez b , J. Roa-Rojas a, se puede encontrar en: https://www.researchgate.net/profile/F_Fajardo/publication/242721297_E structura Electrónica y de Bandas de la Perovskita SnTiO3/links /Odeec528fafdeab72a000000/ Estructura Electrónica y de Bandas de la Perovskita SnTiO3.

C. E. DeLuque, D.A. Landínez Téllez, Jairo Arbey Rodríguez Martínez, J. Roa Rojas, A. Mosquera: https://dialnet.unirioja.es/servlet/articulo?codigo $=5364557$

Volume 50, Issue 358, December 1986, pp. 693-707, https://doi.org/10.1180/minmag.1986.050.358.17

Franco,D.G., Fuertes,V.C., Blanco,M.C., Fernández-Díaz,M.T., Sánchez,R.D., Carbonio, R.E., Synthesis, structure and magnetic properties of La3Co2SbO9: A double perovskite with competing antiferromagnetic and ferromagnetic interactions, J. Solid State Chem. (2012) 194, 385-391

Berlanga Mora Isadora. (2013). Síntesis y caracterización de nanomateriales 0D, 1D y 2D. 2020, de Universidad Autónoma de Madrid Facultad de Ciencias Sitio web: https://repositorio.uam.es/bitstream/handle/10486/660464/berlanga_mora _isadora.pdf?sequence $=1 \&$ is Allowed $=\mathrm{y}$

Glazer,A.M., The Classification of Tilted Octahedra in Perovskites, ActaCryst.(1972)B28

Vazquez Ferreira, Miguel. (2004). Ecuación de Schrödinger. Una aproximación antropológica al conocimiento científico. 2020, de Universidad Complutense de Madrid Sitio web: http://webs.ucm.es/BUCM/tesis//cps/ucm-t27523.pdf

DENSITY FUNCTIONAL THEORY. A Practical Introduction. DAVID S. SHOLL. Georgia Institute of Technology, JANICE A. STECKEL. National Energy Technology Laboratory. Wiley. 2009.

S. Cottenier. Density Functional Theory and the Family of (L)APW-methods: a stepby-step introduction. Ghen University. Pp 87-90. Editorial Belgium. Estados Unidos. 2007.
C. kittel. Introducción a la física del estado sólido. Tercera edición. Cap 2, 4, 7 y 13. Editorial Reverté S.A. Barcelona. 2003

Baúsa, N. (2020). ELECTROLIZADORES DE ALTA TEMPERATURA BASADOS EN CERÁMICAS PROTÓNICAS. València: Consejo Superior De Investigaciónes Cientificas

C. CABALlerO, J. F. FERNANDEZ, P. DURAN, C.. (1994). Titanato de bario cerámico. Departamento de Electrocerámica, Instituto de Cerámica y Vidrio, CSIC, (1), p.5

Parr, Robert G; Yang, Weitao (1989). Density-Functional Theory of Atoms and Molecules Nueva York: Oxford University Press. ISBN 0-19-5042794.Parr, Robert G.; Yang, Weitao (1994). Density-Functional

[23:44, 27/8/2020] Leonardo: Perdew, J. P., Burke, K., \& Ernzerhof, M. (1996). Generalized Gradient Approximation Made Simple. Physical Review Letters, 77(18), 3865-3868. https://doi.org/10.1103/PhysRevLett.77.3865 [23:44, 27/8/2020] Leonardo: Perdew, J. P., Ruzsinszky, A., Tao, J., Staroverov, V. N., Scuseria, G. E., \&

Csonka, G. I. (2005). Prescription for the design and selection of density functional approximations: More constraint satisfaction with fewer fits. Journal of Chemical Physics, 123(6), 062201-2-062201-062209. https://doi.org/10.1063/1.1904565

Monkhorst, H. J., \& Pack, J. D. (1976). Theoretical study on the low-lying excited states of the phosphorus monoiodide (PI) including the spin- orbit coupling. Physical Review B - Condensed Matter and Materials Physics, 13(12), 5188-5192. https://doi.org/10.1016/j.jqsrt.2015.09.006

Arteaga Varela, M., Herrera Carbajal, A. de J., Reyes Valderrama, M. I., \& Rodriguez Lugo, V. (2019). Optimización estructural de la Perovskita de Zirconato de estroncio: un estudio a primeros principios. Pädi Boletín Científico de Ciencias Básicas e Ingenierías Del ICBI, 7(Especial-2), 88-92. https://doi.org/10.29057/icbi.v7iespecial-2.4939

Soler, J. M., Artacho, E., Gale, J. D., García, A., Junquera, J., Ordejón, P., \& Sánchez-Portal, D. (2002). The SIESTA method for ab initio order-N materials simulation. Journal of Physics Condensed Matter, 14(11), 2745-2779. https://doi.org/10.1088/0953-8984/14/11/302.

Khenata, R., Sahnoun, M., Baltache, H., Rérat, M., Rashek, A. H., Illes, N., \& Bouhafs, B. (2005). First-principle calculations of structural, electronic and optical properties of $\mathrm{BaTiO} 3$ and $\mathrm{BaZrO} 3$ under hydrostatic pressure. Solid State Communications, 136(2), 120-125. https://doi.org/10.1016/j.ssc.2005.04.004 